\title{
An oxidative coupling product of luteolin with cysteine ester and its enhanced inhibitory activity for xanthine oxidase
}

Toshiya Masuda, Shoko Nojima, Yukari Miura, Sari Honda, Akiko Masuda

\begin{tabular}{|c|l|}
\hline Citation & Bioorganic \& Medicinal Chemistry Letters, 25(16); 3117-3119 \\
\hline Issue Date & 2015-08-15 \\
\hline Type & Journal Article \\
\hline Textversion & author \\
\hline & $\begin{array}{l}\text { C } 2015 \text { Elsevier Ltd. This manuscript version is made available under the } \\
\text { CC-BY-NC-ND 4.0 License. http://creativecommons.org/licenses/by-nc-nd/4.0/. } \\
\text { Rights }\end{array}$ \\
$\begin{array}{l}\text { This is the accept manuscript version. Please cite only the published version. The } \\
\text { article has been published in final form at } \\
\text { https://doi.org/10.1016/j.bmcl.2015.06.016. }\end{array}$ \\
\hline DOI & \begin{tabular}{l}
$10.1016 / j . b m c l .2015 .06 .016$ \\
\hline
\end{tabular} \\
\hline
\end{tabular}

\author{
Self-Archiving by Author(s) \\ Placed on: Osaka City University
}

MASUDA T, NOJIMA S, MIURA Y, HONDA S, \& MASUDAA. (2015). An oxidative coupling product of luteolin with cysteine ester and its enhanced inhibitory activity for xanthine oxidase. Bioorganic $\&$ Medicinal Chemistry Letters. 25, 3117-9. 


\section{Graphical Abstract}

To create your abstract, type over the instructions in the template box below.

Fonts or abstract dimensions should not be changed or altered.

An oxidative coupling product of luteolin with cysteine ester Leave this area blank for abstract info. and its enhanced inhibitory activity for xanthine oxidase

Toshiya Masuda, Shoko Nojima, Yukari Miura, Sari Honda, and Akiko Masuda
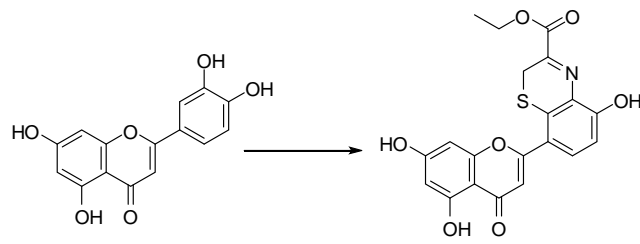

Luteolin (1)

Coupling Product 2

$\mathrm{IC}_{50} 1.7 \mu \mathrm{mol} \mathrm{L}^{-1}$ 


\title{
An oxidative coupling product of luteolin with cysteine ester and its enhanced inhibitory activity for xanthine oxidase
}

\author{
Toshiya Masuda ${ }^{\mathrm{a},{ }^{*}}$, Shoko Nojima $^{\mathrm{a}}$, Yukari Miura ${ }^{\mathrm{b}}$, Sari Honda ${ }^{\mathrm{a}, \mathrm{b}}$, and Akiko Masuda ${ }^{\mathrm{c}}$ \\ ${ }^{a}$ Graduate School of Human Life Science, Osaka City University, Osaka 558-8585, Japan \\ ${ }^{b}$ Graduate School of Integrated Arts and Sciences, University of Tokushima, Tokushima 770-8502, Japan \\ ${ }^{c}$ Faculty of Human Life Science, Shikoku University, Tokushima 770-1192, Japan \\ *Corresponding author, Tel +81-6-6605-2813; E-mail: masuda_t@life.osaka-cu.ac.jp
}

\section{ARTICLE INFO}

\section{ABSTRACT}

Oxidative coupling reactions of several flavonoids with a cysteine ester (a radicalic and nucleophilic biochemical) were carried out and the abilities of the coupling products against xanthine oxidase (XO) were screened. One of the products, derived from luteolin, showed a notable inhibitory effect. A potent XO inhibitory compound was isolated from the complex mixture of the product of the coupling of luteolin and cysteine ethyl ester, and its structure was determined by NMR and MS analysis. The compound has a unique 1,4-thazine ring unit on the luteolin B-ring and is inhibited XO 4.5 times more strongly than it did luteolin.
Keywords:

flavonoid;

xanthine oxidase inhibition;

cysteine:

oxidative coupling;

structure determination
Polyphenols have attracted considerable attention because they have various beneficial effects on human health. ${ }^{1}$ Note that most polyphenols are not very stable under oxidizing conditions. ${ }^{2}$ This ability of polypohenols to be easily oxidized is responsible for their potent antioxidant activities ${ }^{3}$ and for activating cell response systems to oxidative stress. ${ }^{4}$ Chemically, oxidation of polyphenols produces new products that will have not only different chemical structures but also different functionality. We have recently found that an oxidation product of rosmarinic acid strongly inhibits for tyrosinase $\mathrm{e}^{5}$ and that an oxidation product of resveratrol strongly inhibits lipoxygenase. ${ }^{6}$ The oxidative conversion of polyphenols, particularly a polyphenol with a catechol structure, proceeds via the semiquinone radical and subsequent orthoquinone before the final stable product is formed. Therefore, radicalic or nucleophilic molecules surroundings the polyphenol should react with the oxidizing polyphenols. Such coupling reactions afford new products having new functions.

Recent increases in the occurrence of lifestyle-related diseases are causing serious social problems. Gout has now been recognized as a lifestyle-related disease, although it was historically referred to as "the king of diseases." The number of patients with gout and related hyperuricemia in Japan has increased by a factor of three in the last 20 years because of dietary changes and the increased occurrence of metabolic diseases. $^{7}$ Gout is an inflammatory disease that is caused by elevated concentrations of uric acid crystals in serum. Uric acid

is the final metabolite produced from the catabolism of purine in humans, and it is produced by the oxidation of xanthine and hypoxanthine by a key enzyme, xanthine oxidase (XO). Therefore, potent inhibitors of $\mathrm{XO}$ are believed to be good candidates for treating gout.

Flavonoids are widely distributed polyphenols that are easily obtained from plants. Cysteine is an amino acid found in proteins that is strongly reactive because of its thiol and amino groups. The thiol group has not only potent nucleophilcity but also the potential to form a reactive thiyl radical in the presence of $\beta$ amino or carboxylate anions. ${ }^{8}$ Therefore, cysteine has the ability to react with oxidized polyphenols through both nucleophilic and radicalic reactions at the thiol group.<smiles>CCOC(=O)C1=Nc2c(O)ccc(-c3cc(=O)c4c(O)cc(O)cc4o3)c2SC1</smiles>

Coupling Product 2<smiles>O=c1cc(-c2ccc(O)c(O)c2)oc2cc(O)cc(O)c12</smiles> 
Figure 1 Structures of luteolin and oxidative coupling product with cysteine ester. (Each position numbering is based on that of luteolin)

We carried out oxidative coupling reactions between several flavonoids (kaempferol, quercetin, myricetin, taxifolin, luteolin, morin, and catechin) and cysteine ethyl ester, and the degrees to which the product mixtures that were obtained inhibited XO were determined. The oxidative coupling reaction between a flavonoid and cysteine ester was carried out as follows. An acetonitrile solution comprising flavonoid $\left(1.25 \mathrm{mmol} \mathrm{L}^{-1}\right)$, cysteine ethyl ester hydrochloride $\left(1.25 \mathrm{mmol} \mathrm{L} \mathrm{L}^{-1}\right)$, and triethylamine $(1.25$ mmol L') was prepared, and 2,2-diphenyl-1-picrylhydrazyl (DPPH) powder (4 eq.) was added to the solution (at $23^{\circ} \mathrm{C}$ ) with stirring. The mixture was allowed to stand for several minutes, and passed through a short octadecyl silica gel $(1.5 \mathrm{~mL})$ column to remove the reduced DPPH (DPPH-H). The column was eluted with $1 \%$ acetic acid in a $\mathrm{H}_{2} \mathrm{O}-\mathrm{CH}_{3} \mathrm{CN}$ mixture (between 50:50 and 40:60 v/v). The eluate was collected until just before the DPPH-H was eluted, and was evaporated under a reduced pressure. The abilities of the residues that were obtained to inhibit XO were measured using a previously reported method. ${ }^{9}$ Some flavonoids have been found to inhibit XO. ${ }^{10} \mathrm{We}$ found that $\mathrm{XO}$ was strongly inhibited (> 50\% inhibition) by kaempferol, luteolin, myricetin, and quercetin at concentrations of 33 and 100 $\mu \mathrm{mol} \mathrm{L} \mathrm{L}^{-1}$ (Fig. S1 in supporting information). The oxidation of most of the flavonoids that we examined resulted in a decrease in their abilities to inhibit XO. Only the ability of luteolin (1) to inhibit XO was not decreased when it had been oxidized in the presence of cysteine ester. These results indicate that one or more potent $\mathrm{XO}$ inhibitors were present in the product mixture when luteolin reacted with cysteine ester (Fig. 1).

The product mixture produced by the oxidation reaction between luteolin and cysteine ester was analyzed by HPLC, and one of the several product peaks was dominant. This peak was not observed in the DPPH oxidation product mixture when only luteolin was used as a substrate. These results indicated that the dominant product (2) must play an important role in the inhibition of XO. Next, we isolated $\mathbf{2}$ and determined its structure. A solution of luteolin $(100 \mathrm{mg})$ and one equivalent of cysteine ethyl ester hydrochloride was prepared in $\mathrm{CH}_{3} \mathrm{CN}$, and triethylamine $(50 \mu \mathrm{L})$ and $\mathrm{DPPH}(550 \mathrm{mg})$ were subsequently added to the solution (at $23{ }^{\circ} \mathrm{C}$ ), with stirring. The solution was allowed to stand for several minutes and evaporated to dryness under a decreased pressure. The residue was suspended in ca. 1 $\mathrm{mL}$ of chloroform containing $0.02 \mathrm{~mL}$ of acetic acid, and the resulting suspension was removed by centrifugation at $2000 \mathrm{rpm}$ for $5 \mathrm{~min}$. The supernatant was purified by preparative HPLC using a Cosmosil 5SP-II (250 mm long, $10 \mathrm{~mm}$ i.d.) column, using a mixture of $\mathrm{n}$-hexane and $1 \%$ acetic acid in ethyl acetate $(70: 30 \mathrm{v} / \mathrm{v})$ as the mobile phase at a flow rate of $7 \mathrm{~mL} / \mathrm{min}$. The chromatographic peaks were detected at a wavelength of $350 \mathrm{~nm}$. The eluate that contained a peak that eluted at $28 \mathrm{~min}$ was collected, and this contained compound 2 (30 mg). The HR-ESIMS of $2\left(\mathrm{~m} / \mathrm{z} 412.0474[\mathrm{M}-\mathrm{H}]^{-}\right)$revealed that the molecular formula of 2 was $\mathrm{C}_{20} \mathrm{H}_{14} \mathrm{NO}_{7} \mathrm{~S}$, which indicated that 2 was an equimolar coupling product of luteolin and cysteine ester. One proton signal $\left(\mathrm{H}-2^{\prime}\right)$ was missing from the ${ }^{1} \mathrm{H}-\mathrm{NMR}$ spectrum of 2 compared with the spectrum of luteolin, but all of the other proton signals on the A- and C-rings were almost the same in the spectra of luteolin, indicating that the cysteine reacted with the 2position of the B-ring of luteolin. The detailed structure around the 2-position was deduced from the correlations that were found in the HMBC of 2 (Table 1 and Fig. 2). The results showed that 2 has a unique structure, with a 1,4-thiazine ring formed at the $2^{\prime}$ position via sulfur and at the 3 '-position via nitrogen, as is shown in Fig. 2. Similar thiazine compounds have also identified as a model of an intermediate of pheomelanin biosynthesis by Naoliano et al. ${ }^{11}$ Catechol structure found in luteolin and Dopa may afford these thiazine in the presence of cysteine under oxidation conditions.

Table $1{ }^{13} \mathrm{C}$ and ${ }^{1} \mathrm{H}$ NMR Data for 2 (acetone- $d_{6}, 400 \mathrm{MHz}$ )

\begin{tabular}{|c|c|c|c|}
\hline position & C & $\begin{array}{c}\text { Correlated } \mathrm{H} \text { in } \\
\text { HMBC }\end{array}$ & $\begin{array}{l}\mathrm{H} \text { (mult., } J \text { in } \\
\mathrm{Hz} \text { ) }\end{array}$ \\
\hline 2 & 164.7 & $\mathrm{H}-3, \mathrm{H}-6^{\prime}$ & \\
\hline 3 & 109.3 & & $6.39(\mathrm{~s})$ \\
\hline 4 & 182.7 & $\mathrm{H}-3$ & \\
\hline $4 a$ & 105.2 & H-3, H-6, H-8 & \\
\hline 5 & $163.6^{\mathrm{a}}$ & H-6 & \\
\hline 6 & 100.0 & H-8 & $6.29(\mathrm{~d}, 1.6 \mathrm{~Hz})$ \\
\hline 7 & 165.2 & H- 8 & \\
\hline 8 & 94.7 & H-6 & $6.44(\mathrm{~d}, 1.6 \mathrm{~Hz})$ \\
\hline $8 \mathrm{a}$ & 158.7 & $\mathrm{H}-8$ & \\
\hline $1^{\prime}$ & 122.5 & $\mathrm{H}-3$ & \\
\hline $2^{\prime}$ & 127.8 & H-6', H-3" & \\
\hline $3^{\prime}$ & $157.5^{\mathrm{b}}$ & H-5' & \\
\hline $4^{\prime}$ & $157.5^{\mathrm{b}}$ & H-5', H-6' & \\
\hline $5^{\prime}$ & 113.8 & & $6.97(\mathrm{~d}, 8.8 \mathrm{~Hz})$ \\
\hline $6^{\prime}$ & 132.6 & & $7.73(\mathrm{~d}, 8.8 \mathrm{~Hz})$ \\
\hline $1^{\prime \prime}$ & $163.4^{\mathrm{a}}$ & $1^{\prime \prime}-\mathrm{OC} \underline{\mathrm{H}}_{2}$ & \\
\hline $2^{\prime \prime}$ & 149.0 & $\mathrm{H}-3^{\prime \prime}$ & \\
\hline $3^{\prime \prime}$ & 22.9 & & $3.64(\mathrm{~s})$ \\
\hline $1^{\prime \prime}-\mathrm{OCH}_{2} \mathrm{CH}_{3}$ & 63.0 & 1"- $-\mathrm{OCH}_{2} \mathrm{CH}_{\underline{3}}$ & $4.40(\mathrm{q}, 7.2 \mathrm{~Hz})$ \\
\hline 1"- $-\mathrm{OCH}_{2} \underline{\mathrm{CH}_{3}}$ & 14.4 & $1^{\prime \prime}-\mathrm{OC} \underline{\mathrm{H}}_{2}$ & $1.37(\mathrm{t}, 7.2 \mathrm{~Hz})$ \\
\hline
\end{tabular}

${ }^{a}$ These values are interchangeable. ${ }^{b}$ Both or one side may have this value.

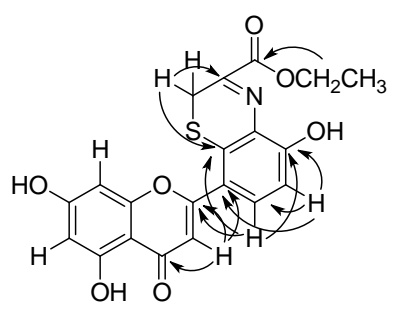

Fig. 2 HMBC correlations around B-ring of 2.

The proposed mechanism through which the unique structure of 2 is produced is illustrated in Fig. 3. First, DPPH abstracts hydrogens from the 3'- or 4'-hydroxy groups because the bond dissociation energies are lower in these groups than in all of the other hydroxyl groups in luteolin. ${ }^{12,13}$ A thiyl radical, which is produced from the reaction between cysteine ester and DPPH, reacts at the 2 '-position, a captodative position, through a very fast radical coupling reaction to produce 5. In an alternative route, quinone $\mathbf{4}$ is produced by an additional equivalent of 
DPPH; then, the cysteine thiol moiety reacts at the $2^{\prime}$-position of the quinone as the nucleophile to produce adduct 5. Although the reason for the selectivity of the reactions occurring at the 2 '- and 5 '-positions is unclear, LUMO of $\mathbf{2}$, which was calculated using MOPAC, indicates that the most reactive positions are the orthopositions of carbonyl groups in quinone 4 (Fig. S2 in supporting information). After the coupling has occurred, the $\alpha$-amino group of the cysteine moiety condenses with the 3'-oxo group of 5 to produce a thiaza ring system, as shown in structure $\mathbf{6}$. The eneamino-alcohol structure seen at the 3'- and 4'-positions of $\mathbf{7}$ is very easily oxidized, so the excess DPPH that is present oxidizes 7 to produce 8, which then gives the stable compound $\mathbf{2}$ as the final product.
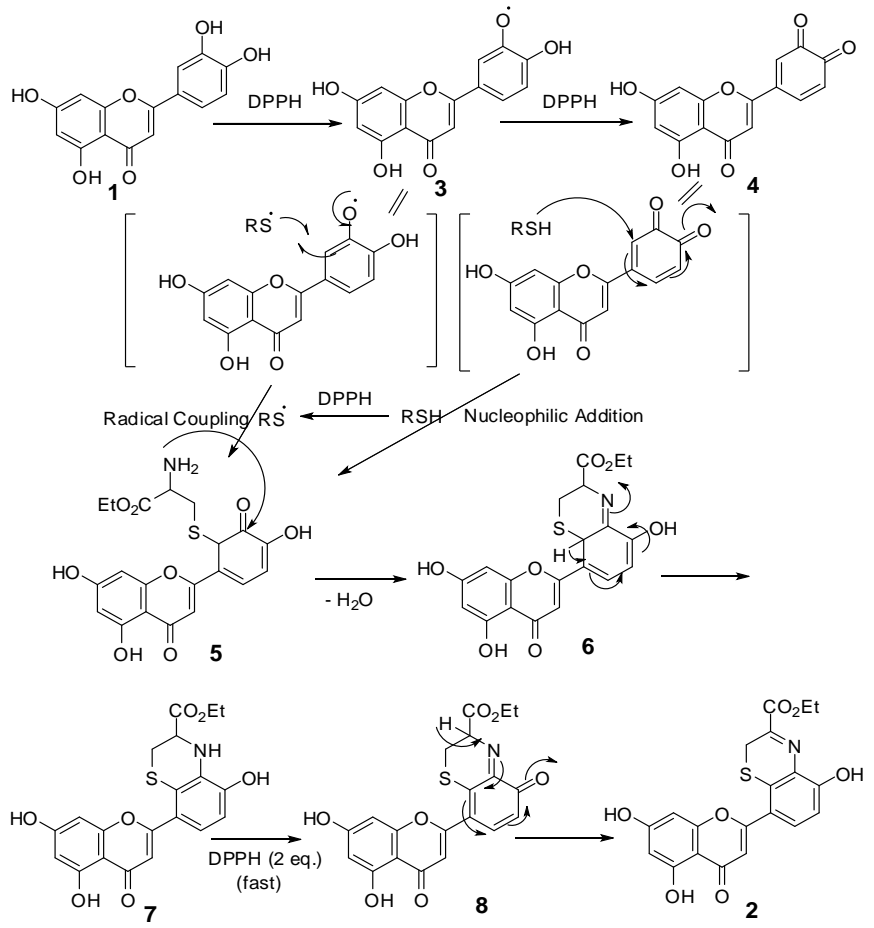

\section{References and notes}

1. Sie, H. Arch. Biochem. Biophys. 2010, 501, 2.

2. Halliwell, B. Arch. Biochem. Biophys. 2008, 476, 107.

3. Frankel, E. N. Lipid Oxidation, second ed., The Oily Press, Bridgwater, 2005

4. Niture, S. K.; Khatri, R.; Jaiswal, A. K. Free Radical Biol. Med. 2014, 66, 36.

5. Fujimoto, A.; Shingai, Y.; Nakamura. M.; Maekawa, T.; Sone, Y.; Masuda. T. Bioorg. Med. Chem. Lett. 2010, $20,7393$.

6. Shingai, Y.; Fujimoto, A.; Nakamura, M.; Masuda, T. J. Agric. Food Chem. 2011, 59, 8180 .

7. Japanese Society for Gout and Nucleic Acid Metabolism, Ed. Guideline for Management of Hyperuricemia and Gout, 2nd ed. 2012 supplement. Medical Review; Osaka, 2012; pp.3-8.

8. Miura, Y.; Honda, S.; Masuda, A,; Masuda, T. Biosci. Biotechnol. Biochem. 2014, 78, 1452.

9. Masuda, T.; Shingai, Y.; Takahashi, C.; Inai, M.; Miura, Y.; Honda, S.; Masuda, A. Free Radical Biol. Med. 2014, 69, 300.

10. Cos, P.; Ying, L.; Calomme, M.; Hu, J. P.; Cimanga, K.; Poel, B. V.; Pieters, L.; Vlietinck, A. J.; Berghe, D. V. J. Nat. Prod. 1998 61,71 .

11. Napolitano, A.; Donato, P. D.; Prota, G. J. Org. Chem. 2001, 66 , 6958.

12. Amić, D.; Lučić, B. Bioorg. Med. Chem. 2010, 18, 28.

13. Leopoldini, M.; Pitarch, I. P.; Russo, N.; Toscano, M.; J. Phys. Chem. A. 2004, 108, 92.

14. López-Lázaro, M. Mini-Rev. Med. Chem. 2009, 9. 31.

15. Odontuya, G.; Hoult, J. R. S.; Houghton, P. J. Phytother. Res. 2005, 19, 782 .

16. Zang, J.; Liu, X.; Lei, X.; Wang, L.; Guo, L.; Zhao, G.; Lin, G. Bioorg. Med. Chem. 2010, 18, 7842.
Fig. 3. Proposed formation mechanism of 2

The ability of $\mathbf{2}$ to inhibit XO was determined using a previously reported procedure. ${ }^{9}$ Compound 2 inhibited XO in a concentration-dependent manner at between 1 and $10 \mu \mathrm{mol} \mathrm{L}-1$, and its $\mathrm{IC}_{50}$ was found to be $1.7 \mu \mathrm{mol} \mathrm{L} \mathrm{L}^{-1}$. Luteolin is recognized as being a strong $\mathrm{XO}$ inhibitor that is found in various medicinal herbs, ${ }^{10}$ and using our method, its $\mathrm{IC}_{50}$ value was found to be 7.8 $\mu \mathrm{mol} \mathrm{L}{ }^{-1}$. Therefore, the coupling reaction between luteolin and cysteine ethyl ester produced a product that inhibited XO 4.5 times more effectively than luteolin.

Luteolin is a widely distributed flavonoid in the plant kingdom, and it has been found to have various biological activities, including as an XO inhibitor, an antioxidant, an antiinflammatory agent, and a chemopreventive agent for cancer treatments. ${ }^{14}$ Expectations of improving on the various beneficial activities of flavonoids themselves have prompted medicinal chemists to produce many derivatives of flavonoids. Derivatives of luteolin that have been synthsized have included glycosides for use as anti-inflammatory agents ${ }^{15}$, and alkyl ethers for use as dopamine transporter activators ${ }^{16}$ and antibacterial agents. ${ }^{17}$ An oxathiolane derivative of quercetin was found to inhibit XO more than quercetin itself. ${ }^{18}$ In this communication we have reported a new luteolin derivative that inhibits XO more effectively than luteolin itself. The derivative is formed through an oxidative coupling reaction, and this could be a useful method for preparing efficient bioactive derivatives of polyphenolic flavonoids.

\section{Acknowledgments}

This investigation was financially supported by JSPS Kakenhi (Grant No. 15H02892).

17. Lv, P.-C.; Li, H,-Q.; Xue, J.-Y.; Shi, L.; Zhu, H.-L. Eur. J. Med Chem. 2009, 44, 908.

18. Takahama, U.; Koga, Y.; Hirota, S.; Yamauchi, R. Food Chem. 2011, 126, 1808 